\title{
MAGNETIC FIELD DEPENDENCE OF QUASI-BOUND COULOMB STATES OF LIGHT HOLES IN GERMANIUM
}

\author{
G. Jungwirt, R. Kropf, U. Roessler and W. Prettl \\ Fakultät für Physik der Universität Regensburg \\ D-8400 Regensburg, W-Germany
}

(Received July 4, 1989 by M. Cardona)

\begin{abstract}
The photothermal spectra of the shallow acceptor boron in high purity $\mathrm{p}$-Ge has been investigated for various magnetic field strengths at low temperatures. A large number of lines observed at frequencies above the binding energy of boron are attributed to optical excitation of quasi-bound Coulomb states, which arise in a magnetic field below each Landau level. By comparison with calculated Landau level energies the final states of the observed transitions can be unambigously identified.
\end{abstract}

Shallow impurities in semiconductors represent hydrogen-like systems for which the high magnetic field case can be exprimentally realized by standard laboratory magnets due to the large effective Bohr radius. Therefore the optical excitation spectra of shallow impurities in a uniform magnetic field have been extensively studied by far-infrared (FIR) photothermal ionization and photoionization spectroscopy [1]. The hydrogen atom in a magnetic field is a fundamental problem of quantum theory, which cannot be solved in closed form because of different symmetries of the Coulomb potential and the uniform magnetic field. The energy spectrum of this system consists besides of continuum states, corresponding to Landau levels with quantum number $n$, of bound (stable) or quasi-bound (metastable) Coulomb states depending on their angular momentum quantum number $\mathrm{m}[2,3]$. Due to the relation $m \leq n$, the Coulomb states connected with the higher Landau levels $n \geq 1$ and $m \leq n-1$ are degenerate with the continuum of lower Landau levels with the same $m$ quantum number, to which they are coupled by the Coulomb potential. Thus autoionization transitions may occur and, therefore, these states are called quasi-bound or metastable. In contrast, the Coulomb states connected with the lowest Landau level $n=0$ and $m \leq 0$ and those with $n=m$, which are not degenerate with a continuum, are bound or stable.

In a previous investigation of shallow donors in nGaAs several structures in FIR photoconductivity spectra could be attributed to optical transitions from the donor ground state to those metastable states [4]. The identification of the final states was based on the fact that pairs of states with $m= \pm 1$ separated by the cyclotron resonance energy $\hbar w_{c}$ are optically accessible in Faraday configuration from the donor ground state using unpolarized light. In the fourfold degenerate valence band of tetrahedrally coordinated semiconductors the situation is more complex because the Landau levels are irregularly spaced [5]. Zverev et al. analyzed p-Ge in detail by investigating magneto- photoconductivity upon monochromatic irradiation [6] and optical phonon emission induced resonant recombination of holes excited by broad band infrared illumination [7]. For each degenerate Landau level they observed a single resonance attributed to quasi-bound acceptor states.

In the present paper we report on FIR photoconductivity measurements on boron doped p-Ge carried out by Fourier-spectroscopy. In high purity samples a whole series of spectral lines has been observed and identified from their magnetic field dependence as being attached to the light hole Landau levels. Each line itself exhibits fine structure which may be resolved by high resolution laser magneto-spectroscopy.

Measurements were performed on a p-Ge sample containing the shallow acceptors boron and aluminium with a density ratio $n_{B} / n_{A l}=10$ and an effective acceptor concentration of $2.5 \times 10^{12} \mathrm{~cm}^{-3}$. The compensation ratio was $1 \%$. Ohmic contacts were made on the sample by alloying $\mathrm{Al}$ on opposite edges. The sample was mounted at the end of a metallic light pipe fitted in a variable temperature cryostat in the center of a superconducting magnet. The photoconductivity spectra were measured in Faraday configuration with the magnetic field normal to the [111] faces of the crystal, using a commercial far-infrared Fourier- spectrometer in the frequency range between 45 and $200 \mathrm{~cm}^{-1}$. A standard load resistor circuit was used and the bias voltage was kept well below the impact ionization threshold of shallow acceptors to avoid impact ionization nonlinearities [8].

The photoconductivity spectrum at zero magnetic field obtained at a temperature of $7.5 \mathrm{~K}$ is shown in Fig. 1 (a) in a frequency range between 45 and $200 \mathrm{~cm}^{-1}$. The observed lines are due to photothermal ionization caused by transitions from the $1 S_{3 / 2}\left(\Gamma_{8}^{+}\right)$acceptor 
ground state to excited states below the valence band edge [9]. The labelling of the lines in Fig. 1 is according to Jones et al. [10]. From the well known spectral pattern of the zero magnetic field lines the involved acceptors can be identified [11]. The present sample contains predominantly the shallow acceptor boron. Above the binding energy of boron of about $87 \mathrm{~cm}^{-1}$ a continuous photospectrum is observed caused by direct photoionization of impurities without involving a thermal phonon.
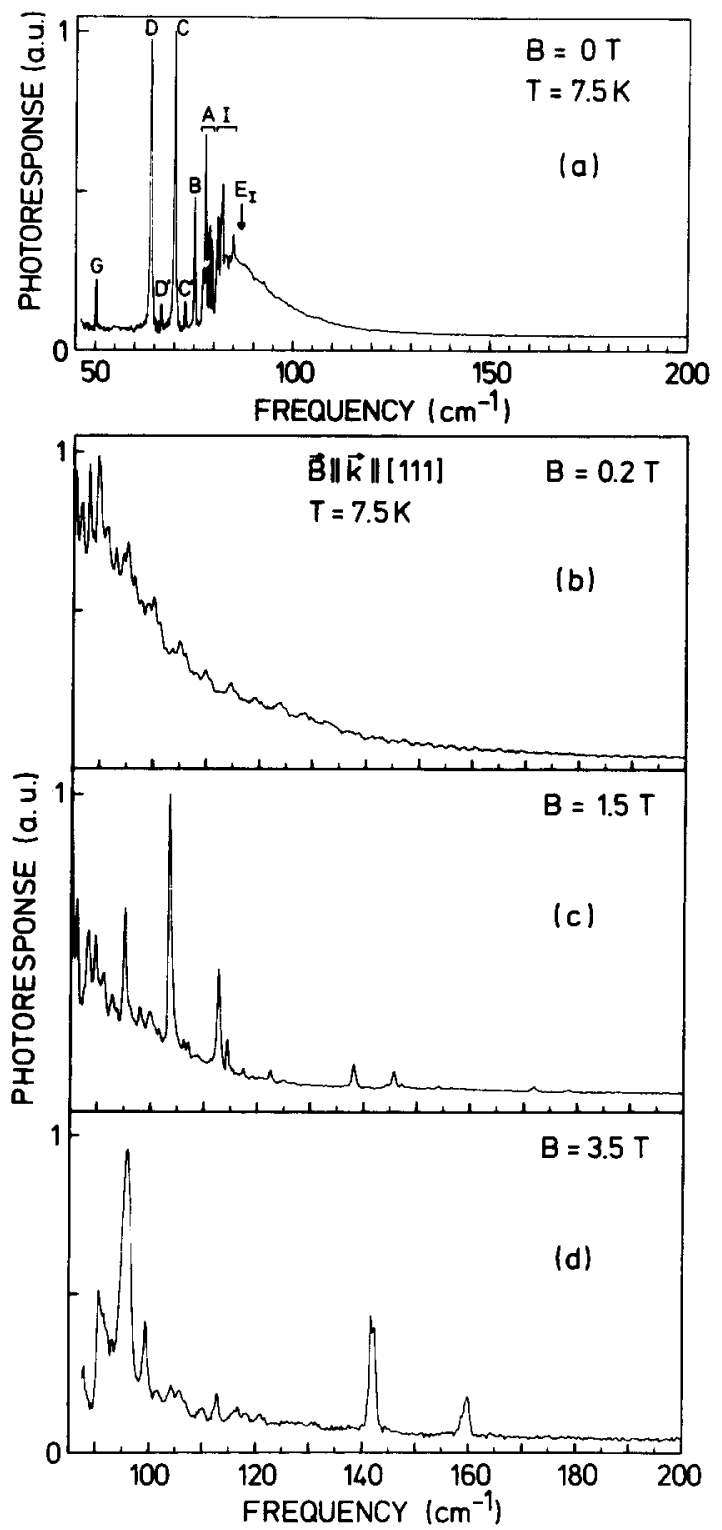

Figure 1: Photoconductivity spectra of $\mathrm{p}-\mathrm{Ge}$ for various magnetic field strengths. (a) $B=0$ : labelling after [9]. The lines are due to boron and aluminium (primed). (b)-(d) $B>0$ : Faraday configuration with the incident beam parallel to [111] direction : the spectra show the emergence of sharp structures in the continuum with increasing $B$.
At $B=0 T$ the photoionization continuum is a smooth curve without any pronounced spectral structure.

In Fig. 1 (b) - (d) photoconductivity spectra are shown for three different magnetic field strength up to $3.5 \mathrm{~T}$ in a frequency range above the threshold of di-

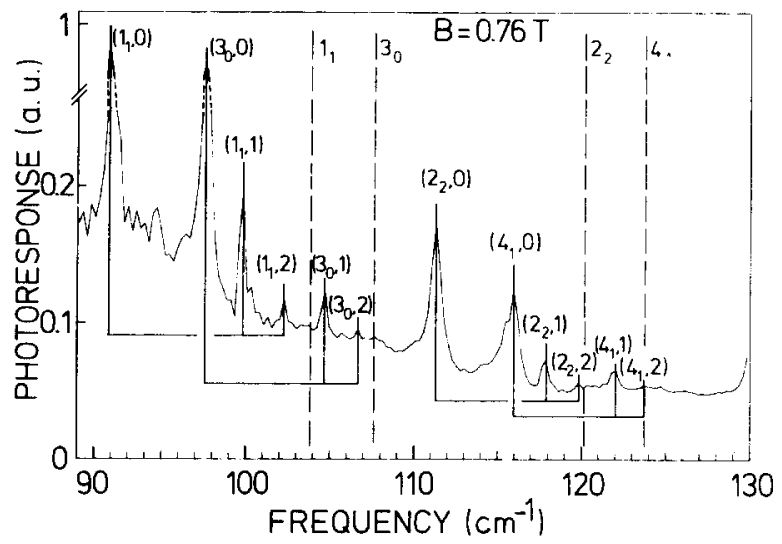

Figure 2: A detail of the spectrum at $B=0.76 \mathrm{~T}$. The three strongest lines belonging to one series are indicated by bars on a common base line and labelled by quantum numbers $\left(N_{n_{a}}, \kappa\right)$.
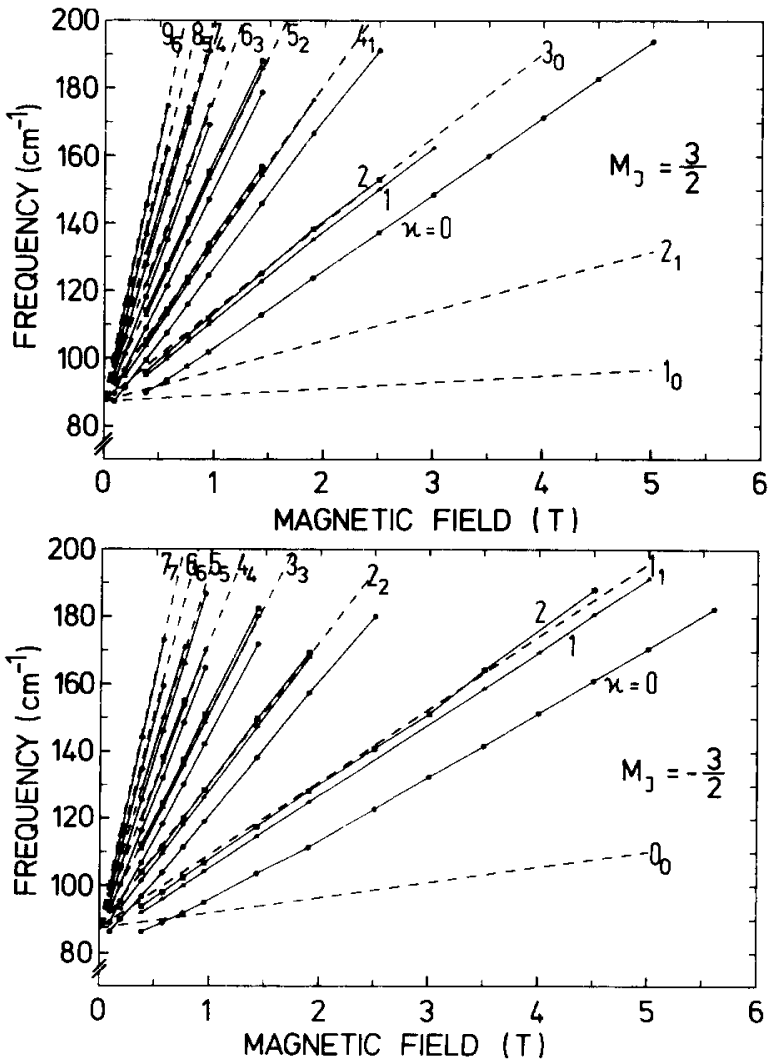

Figure 3: Magnetic field dependence of all lines observed in the present study. Broken lines show Landau levels with respect to the $B=0$ ionization energy of boron acceptor. 
rect photoionization. With increasing magnetic field strength sharp spectral structures emerge out of the continuum. Even at a field strength as small as $0.2 \mathrm{~T}$ a modulation of the continuum can clearly be observed. At larger magnetic fields pairs of strong lines dominate the spectra. They shift with increasing magnetic field to higher energies similar to the light hole Landau levels [12]. A careful analysis of the spectra shows that each strong line is connected with a series of weaker lines at higher energies (Fig. 2). In the limit of accuracy of the present measurements up to three lines of each series could uniquely be identified, which in Fig. 2 are connected by bars and labelled by $\left(N_{n_{a}}, \kappa\right)$. $N=n_{a}+M_{j}+3 / 2$ is the usual notation of Landau levels in spherical approximation which combines the oscillator quantum number $n_{a}$ with the Bloch state angular momentum $M_{j}[5,12] . \kappa$ numbers the Coulomb states by counting the nodes of the envelope wave function on an axis parallel to the magnetic field [2].

In Fig. 3 the peak positions of all observed line series for magnetic field strengths up to $5.6 \mathrm{~T}$ are plotted as a function of the magnetic field strength. For the sake of clearness resonances belonging to the $M_{j}=+3 / 2$ and $M_{j}=-3 / 2$ Landau level ladders are presented separately. $M_{j}$ is the angular spin momentum quantum number of holes. The broken lines represent the energy of calculated Landau levels [12] relative to the zero magnetic field ground state binding energy of $87 \mathrm{~cm}^{-1}$. These curves serve as guide lines for identification of the observed resonances. As the magnetic field dependence of the acceptor ground state is not known, the correct energy separation between Landau levels and the $1 S_{3 / 2}$ $\left(\Gamma_{8}^{+}\right)$state cannot be plotted. Generally the ground state, mainly constituted by heavy hole contributions, is expected to shift slightly to lower energies with increasing magnetic field. Thus the continuum threshold of each Coulomb resonance series must be somewhat above the broken lines in Fig. 3 .

Each line itself shows fine structure which is not well resolved in the Fourier transform spectra. Some indication of line splitting is seen for the line $\left(1_{1}, 0\right)$ in Fig. 1 (d) at $\mathrm{B}=3.5 \mathrm{~T}$. This result is supported by laser magneto-spectroscopy measurements, which will be published elsewhere.

For a more detailed understanding of the observed spectra we look at the energy level diagram of the Landau levels together with the Coulomb states. In Fig. 4 we plot the acceptor ground state and excited states connected with each one Landau level with $M_{j}=+3 / 2$ $\left(3_{0}\right)$ and $M_{j}=-3 / 2\left(1_{1}\right)$ and different $m$. The Coulomb states bear the additional quantum number $\kappa$. The total angular momentum parallel to the magnetic field $m=M_{j}+m_{l}$ is composed of the Bloch state angular momentum $M_{j}$ and the orbital angular momentum $m_{l}=n_{a}-n_{b}$ [13]. Metastable states are those with $m+3 / 2<N$. The second oscillator quantum number $n_{b}[5]$ counts the degeneracy of the Landau levels due to the translational invariance perpendicular to the magnetic field. This degeneracy is lifted in the presence of impurities, which break the translational symmetry.

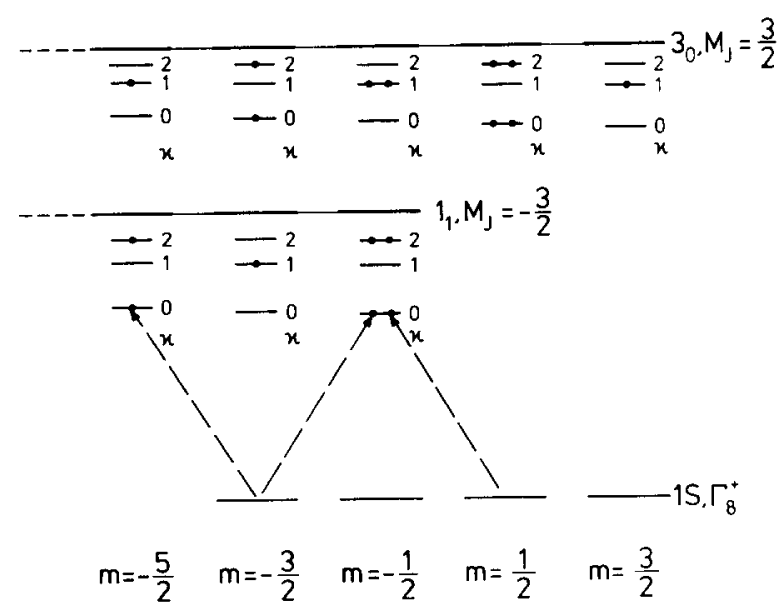

Figure 4: Schematic energy level diagram including the acceptor ground states, the $1_{1}$ and $3_{0}$ Landau levels and quasi-bound states. Final states accessible by electric dipole transitions from the ground state are indicated by dots and for the lowest levels, by arrows demonstrating the general structure of the spectra.

Therefore, the metastable states are expected to depend on $n_{b}$ and thus on $m_{l}$ with decreasing binding energies for increasing $n_{b}$ [2]. The acceptor ground state $1 S_{3 / 2}$ $\left(\Gamma_{8}^{+}\right)$is split into four levels with $M_{j}=-3 / 2 \ldots+3 / 2$ in a magnetic field. Actually the energy separation of these levels is vanishingly small $[14,15]$, definitely smaller than the observed line splittings. Thus the fine structure of the resonances must be due to different final state energy levels.

The electric dipole selection rules for Faraday configuration and spherical approximation are $\Delta m= \pm 1$ and $\pi_{i} \pi_{f}=-1$ where $\pi_{i, f}$ are the parity of the acceptor ground state, $\pi_{i}=+1$, and the excited state, respectively. The parity is given by $\pi=(-1)^{\left(n_{a}+n_{b}+\kappa\right)}$. These selection rules yield allowed optical transitions to final states marked in Fig. 4 by dots and indicated by arrows for the lowest excited states. Thus, as observed, we expect a series of lines with increasing frequency below each degenerate Landau level instead of one sharp transition as found previously. The fine structure of the lines is attributed to energetically close lying final states of different $\mathrm{m}$ but having the same $\kappa$. Two transitions are degenerate yielding one line. Thus we expect a fine structure of two lines. The spherical approximation, which has been used to simplify the qualitative discussion, is of limited accuracy. Indeed the cubic contributions to the potential cannot be neglected as is obvious from the zero field structure of the spectrum. This reduction of symmetry mainly breaks the $\Delta m$ selection rule allowing more transitions. Thus the fine structure may have even more then two components.

In summary, optical excitations of holes from the $1 S_{3 / 2}\left(\Gamma_{8}^{+}\right)$ground state of the boron acceptor to metastable or quasi-bound Coulomb states have been de- 
tected by photoconductivity measurements of $\mathrm{p}-\mathrm{Ge}$ in a magnetic field. Series of up to three lines connected with each light hole Landau level are identified belonging to states with different $\kappa$. Each line has a fine structure, which is due to relaxation of the translational symmetry of the cyclotron orbit due to the magnetic field.
Acknowledgements - We thank E.E. Haller for provision of the germanium crystal. Financial support by the Deutsche Forschungsgemeinschaft is gratefully acknowledged.

\section{REFERENCES}

[1] A.K. Ramdas and S. Rodriguez, Rep. Prog. Phys. 44, 1297 (1981).

[2] J. Simola and J. Virtamo, J. Phys. B 11, 3309 (1978).

[3] V.F. Gantmakher, B.L. Gelmont, V.N. Zverev and Al.L. Efros , Sov. Phys. JETP 57, 656 (1983).

[4] H.P. Wagner and W. Prettl, Solid St. Commun. 66, 367 (1988).

[5] K. Suzuki and J.C. Hensel, Phys. Rev. B 9, 4184 (1974).

[6] V.N. Zverev, Sov. Phys. Solid State 22, 1921 (1980).

[7] V.F. Gantmakher and V.N. Zverev, Sov. Phys. JETP 52, 1159 (1980).

[ 8] E. Schöll, W. Heisel and W. Prettl, Z. Phys. B 47, 285 (1982).
[9] N.O. Lipari and A. Baldereschi, Solid St. Commun. 25, 665 (1978).

[10] R.L. Jones and P. Fisher, J.Phys. Chem. Solids 26, 1125 (1965).

[11] E.E. Haller and W.L. Hansen, Solid St. Commun. 15, 687 (1974).

[12] H.-R. Trebin , U. Rössler and R. Ranvaud, Phys. Rev. B 20, 686 (1979).

[13] As usual in literature we use single electron quantum numbers instead of hole quantum numbers which are related by time inversion to the corresponding states.

[14] H. Tokumoto and T. Ishiguro, Phys. Rev. B 15, 2099 (1977).

[15] C.A. Freeth , P. Fisher and P.E. Simmonds , Solid St. Commun. 60, 175 (1986). 